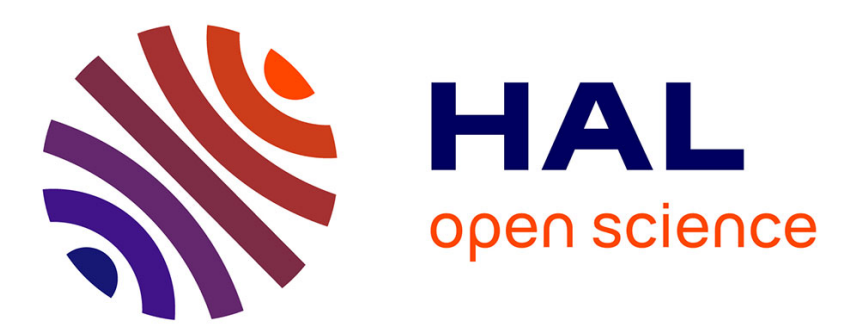

\title{
Advanced simulation of CBRAM devices with the level set method
}

A C Dorion, A C Cueto, A C Reyboz, A C Barbé, A C Grigoriu, Yvon Maday

\section{To cite this version:}

A C Dorion, A C Cueto, A C Reyboz, A C Barbé, A C Grigoriu, et al.. Advanced simulation of CBRAM devices with the level set method. SISPAD , Sep 2014, Yokohama, Japan. pp.33 - 36, 10.1109/SISPAD.2014.6931556 . hal-01476528

\section{HAL Id: hal-01476528 \\ https://hal.science/hal-01476528}

Submitted on 24 Feb 2017

HAL is a multi-disciplinary open access archive for the deposit and dissemination of scientific research documents, whether they are published or not. The documents may come from teaching and research institutions in France or abroad, or from public or private research centers.
L'archive ouverte pluridisciplinaire HAL, est destinée au dépôt et à la diffusion de documents scientifiques de niveau recherche, publiés ou non, émanant des établissements d'enseignement et de recherche français ou étrangers, des laboratoires publics ou privés. 


\title{
Advanced simulation of CBRAM devices with the level set method
}

\author{
P. Dorion ${ }^{1,2}$, O. Cueto ${ }^{1}$, M. Reyboz ${ }^{1}$, J.C. Barbé ${ }^{1}$, A. Grigoriu ${ }^{3}$, Y. Maday ${ }^{2}$ \\ ${ }^{1}$ CEA-LETI, Campus MINATEC, 17 rue des Martyrs, 38054 Grenoble, Cedex 9, France \\ ${ }^{2}$ UPMC Univ. Paris 6, UMR 7598, J.-L Lions Laboratory, F-75005, Paris, France \\ ${ }^{3}$ Univ. Paris Diderot, Sorbonne Paris Cité, J.-L Lions Laboratory, UMR 7598, UPMC, CNRS, F-75205, Paris, France \\ e-mail: pierre.dorion@cea.fr
}

Introduction: Conductive-Bridge Random-Access memory (CBRAM) is a promising technology for future nonvolatile memories, due to its low operating voltages, low power consumption and ease of integration in the back end of a logic process. A CBRAM cell is composed of a resistive switching layer encapsulated between an electrochemically active electrode, and an electrochemically inert counter electrode. The storage of the information is based on the contrast between a high resistance state and a low resistance state. Resistance switching is induced by electro-chemical driven growth and rupture of a metallic filament in the electrolyte. During write operation (SET), cations obtained from the oxidation of the top electrode migrate through the electrolyte and are reduced on the filament contributing to its growth. Starting from an existing model [1], we improve the numerical implementation of the level set method. The physical model is significantly improved by taking into account nucleation and presence of clusters in the electrolyte.

Physical model: We study a CBRAM cell based on an active Ag top electrode, a $\mathrm{GeS}_{2}$ electrolyte and a $\mathrm{W}$ inert electrode.

Oxidation reaction is modeled with a Butler-Volmer equation:

$$
J_{B V}=j_{0}\left[\exp \left(\frac{\alpha e \eta}{k_{B} T}\right)-\exp \left(-\frac{(1-\alpha) e \eta}{k_{B} T}\right)\right]
$$

where $\eta$ is the overvoltage, $k_{B}$ is the Boltzmann constant, $\mathrm{T}$ is the temperature and $e$ is the elementary charge.

The initialization of the filament is obtained with the nucleation model presented by Milchev [2]. The nucleation rate is modeled through the equation:

$$
J_{\text {nuc }}=C\left(Z_{0}, N_{\text {crit }}\right) \exp \left(-\frac{N_{\text {crit }} e\left|\eta_{n u c}\right|}{2 k_{B} T}\right)
$$

where $\eta_{\text {nuc }}$ is the electrochemical overpotential. The parameter $C\left(Z_{0}, N_{\text {crit }}\right)$ depends on the nucleation sites number density $Z_{0}$ and the number of atoms in a critical nucleus $N_{\text {crit }}$. Nucleation is implemented as a stochastic process on the bottom electrode. Filament growth from critical nuclei is modeled using Faraday's law. SET operation is modeled through coupled equations:

$$
\begin{gathered}
e \frac{\partial C_{A g^{+}}}{\partial t}-\operatorname{div}\left(e D \nabla C_{A g^{+}}+\sigma \nabla V\right)=\int_{\Omega} f \mathbf{1}_{b d} \\
-\operatorname{div}(\epsilon \nabla V)=e C_{A g^{+}}
\end{gathered}
$$

The equation (3) models the ionic concentration conservation in the electrolyte and the Poisson's equation (4) couples the electric field and the local ionic concentration. The parameters $V, C_{A g^{+}, \epsilon, \sigma}$ and $\mathrm{D}$ represent respectively the electric potential, the concentration of $\mathrm{Ag}^{+}$, the permittivity, the conductivity and the coefficient of diffusion of $\mathrm{Ag}^{+}$. The $\mathrm{Ag}^{+}$reduction at surface filament is taken into account through a source term included in (3) with $f=S_{T E} / S_{r e d} J_{B V}$. The terms $S_{T E}$ and $S_{r e d}$ represent respectively the surface of the anode and the surface of the reduction area (Fig.1). The function $\mathbf{1}_{b d}$ is an interface filamentelectrolyte indicator function.

In $\mathrm{GeS}_{2}$ electrolytes, Ag-rich clusters, known to be good ionic conductors, are observed experimentally [3]-[4]. Our model is extended by taking into account several clusters in the electrolyte. We consider that if a filament reaches a cluster, the local ionic flux increases the silver concentration inside the cluster. When a sufficient silver concentration is obtained, the cluster electrical properties $(\epsilon, \sigma)$ equal those of the filament [5]; cluster becoming an electron supplier. Nevertheless clusters and filament chemical composition remain different and nucleation step is needed allowing filament growth to restart.

Physical model numerical implementation Level Set Method (LSM) introduced by Osher and Sethian [6] is a versatile method used in many problems with an interface motion [7]. The filament is an area inside the electrolyte with different physical properties, so we use a space-time function $\varphi$ to follow filament growth. Initialization of $\varphi$ is obtained through the nucleation model applied on the bottom electrode. In our model, $\varphi$ ranges between 0 and 1 . The interface between filament and electrolyte is defined by the level set $\varphi=0.5$. The level set function $\varphi$ is solution of an advection equation:

$$
\frac{\partial \varphi}{\partial t}+\vec{v} \cdot \nabla \varphi=0
$$

where $\vec{v}$ represents the velocity of filament's growth.

When a filament reaches a cluster, cluster and filament are merged as an extension of the bottom electrode. The level set function $\varphi$ is reinitialized from a nucleation step which occurs at the cluster surface.

Level Set Method stabilization scheme: In order to stabilize the approximation of the solution of equation (5), we adapt a stabilization scheme previously proposed by Olsson [8]:

$$
\frac{\partial \varphi}{\partial \tau}+\operatorname{div}(\varphi(1-\varphi) \vec{n})=\xi \Delta \varphi
$$

where the normal at the interface $\vec{n}$ is solution of :

$$
\vec{n}-\chi \Delta \vec{n}=\frac{\nabla \varphi}{\|\nabla \varphi\|}
$$

The normal at the interface presents numerical instabilities, so the diffusive term in equation (5.b) is adjusted to regularize the normal near the level set $\varphi=0.5$. As recommended by Olsson [8], we take $\xi=h$, where $h$ is the characteristic mesh size. The choice of $\chi=10^{-17}$ results from numerical tests.

The nonlinear hyperbolic equation (5.a) is discretized in:

$$
\begin{aligned}
\frac{\varphi_{k+1}^{n}-\varphi_{k}^{n}}{\Delta \tau}+\operatorname{div} & \left(\frac{\varphi_{k+1}^{n}+\varphi_{k}^{n}}{2}-\varphi_{k+1}^{n} \varphi_{k}^{n}\right) \\
& =\epsilon \nabla\left(\frac{\varphi_{k}^{n}+\varphi_{k+1}^{n}}{2}\right) \cdot \vec{n} \operatorname{div}(\vec{n})
\end{aligned}
$$

In order to stabilize the solution $\varphi^{n}$ at each time step, we implement equation (5.c) in an iterative scheme.

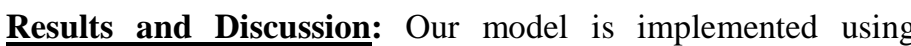
software FreeFem++ [9]. Equations (3), (4), (5), (5.a) and (5.c) are discretized using Lagrange finite element on a cartesian mesh. The constants used in simulations are given in Tab.1. We simulate the system in a $2 \mathrm{D}$ domain corresponding to perpendicular cut plane of the inert electrode in a 3D structure (Fig. 1).

First, we study the stabilization effect of equations (5.a) and (5.c) neglecting clusters contribution. The value of the stabilization parameters $\chi$ and $\xi$ are fixed by this study. Figure 2 illustrates the efficiency of stabilization scheme after few time steps. Please note that for long physical time, branched filament topology appears (Fig.2d-e).

Second, we introduce clusters in the electrolyte. When the nucleation step is activated to reinitialize $\varphi$, we obtain one or several critical nuclei where electrical field is stronger (Fig.3a). From each nucleus, our model succeeds to restart filament in the electrolyte (Fig.3b). The relative permittivity in the filament is chosen large enough to model electrical neutrality inside the filament (Fig.4). The ionic conductivity and diffusivity inside cluster show constant silver concentration inside the clusters (Fig.5). In a 2D domain with clusters, metallic filament can connect clusters before reaching the top electrode (Fig.6).

Conclusion and Perspectives: The nucleation and filament growth in a CBRAM device containing clusters can be simulated with our model. We improve the level set method implementation by adjusting the normal at the interface. We will study the branched topology origin. We improve the physical model by taking into account nucleation and Ag-rich clusters in 
the electrolyte. The influence of clusters on CBRAM electrical characteristics (SET resistance, switching time ...) will be studied. Thanks to the introduction of nucleation in our model, we will be able to study which phenomenon, between nucleation, cations migration and redox reaction, is the rate limiting step in switching time. Extension to 3D simulation would be natural because the level set method extends naturally to 3D.

Acknowledgments: The authors would like to thank Frédéric Hecht, Charles Dapogny and Pascal Frey for fruitful discussion and for sharing FreeFem++ experience.

\section{Reference}

[1] P.Dorion et.al., SISPAD Proceeding, pp. 340-343-2663 (2013)

[2] A.Milchev, Electrocrystallization, (2002)

[3] V.Sousa, Microelec. Engineering, 88 pp. 807-813 (2011)

[4] M.N.Kozicki et.al., Superlattices and Micro, 34 pp. 459-465 (2003)

[5] Z.Tsanka et.al., ESSDERC 2013

[6] S.Osher et.al., S. J. Comp. Physics, 79, pp. 12-49 (1988)

[7] M.F.Gyure et.al., M.-F. Phys. Review, 58 (6), pp. 6927-6930 (1998)

[8] E.Olsson et.al., E. J. Comp. Physics, 225, pp. $785-807$ (2007)

[9] F.Hecht, J.Numer.Math., 20, pp. 251-265 (2012)

\begin{tabular}{|c|c|c|c|}
\hline \multicolumn{4}{|c|}{ Table 1. Parameters used in the simulation } \\
\hline Parameter & Value & Parameter & Value \\
\hline$\alpha$ & 0.154 & $\mathrm{j}_{0}$ & $10^{-2} \mathrm{~A} \cdot \mathrm{m}^{-2}$ \\
\hline$N_{\text {crit }}$ & 8 & $\Delta \tau$ & $10^{-10} \mathrm{~s}$ \\
\hline$\epsilon_{\text {fil }}$ & $10^{6} \epsilon_{0}$ & $\epsilon_{\text {elec }}$ & $7 \epsilon_{0}$ \\
\hline$D_{\text {fil }}$ & $10^{2} \mathrm{~m}^{2} \cdot \mathrm{s}^{-1}$ & $D_{\text {elec }}$ & $10^{-3} \mathrm{~m}^{2} \cdot \mathrm{s}^{-1}$ \\
\hline
\end{tabular}

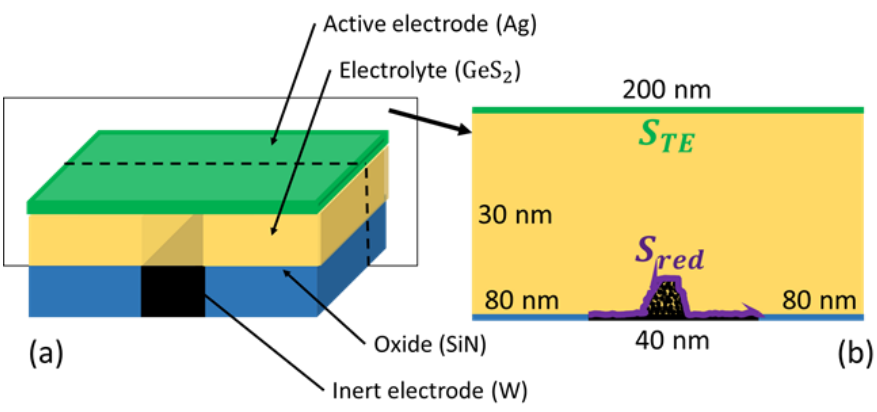

Figure 1. (a) 3D representation of study device, (b) 2Ddomain of resolution with the representation of $S_{r e d}$ and $S_{T E}$.
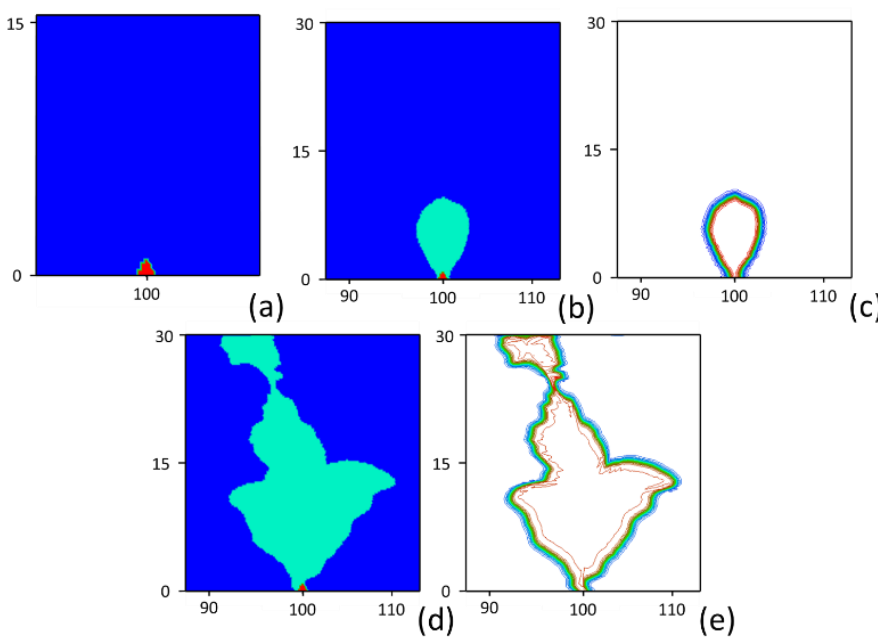

(Lengths are in nanometer)

Figure 2. Results of advection equation simulation with stabilization scheme and no clusters. (a) Initial Filament, (b) Filament at time t=700 ns, (c) $\varphi$ at time $\mathrm{t}=700 \mathrm{~ns}$, (d) Filament at time $\mathrm{t}=1.8 \mu \mathrm{s}$, (e) $\varphi$ at time $\mathrm{t}=1.8 \mu \mathrm{s}$
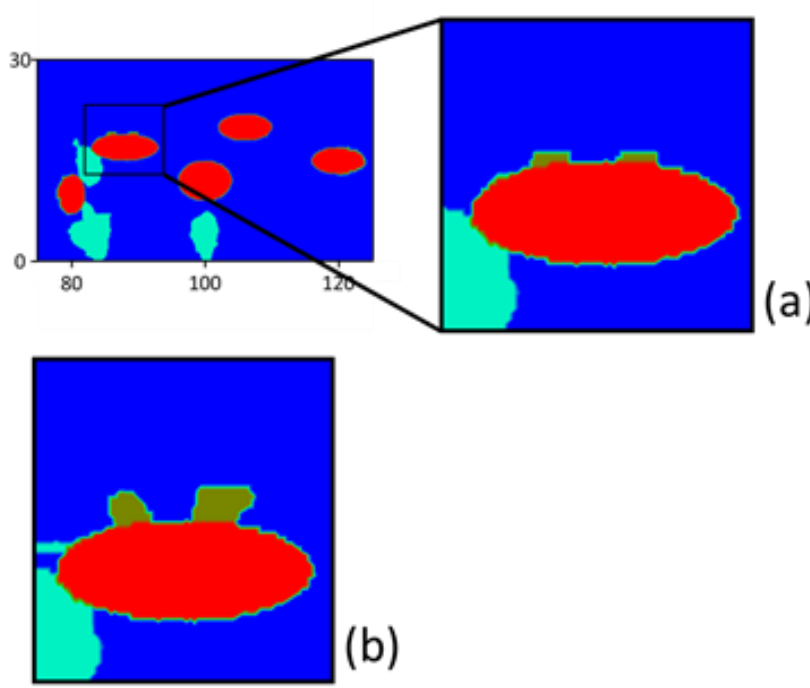

$$
\epsilon=\epsilon_{\text {int }}(\varphi>0.5)+\epsilon_{\text {elec }}(\varphi<0.5)
$$

Figure 3. (a) Reinitialization of $\varphi$ after a nucleation step implied by clusters electrical properties changes at time $\mathrm{t}=1.1 \mu \mathrm{s}$. (b) Filament at time $\mathrm{t}=1.15 \mu \mathrm{s}$.

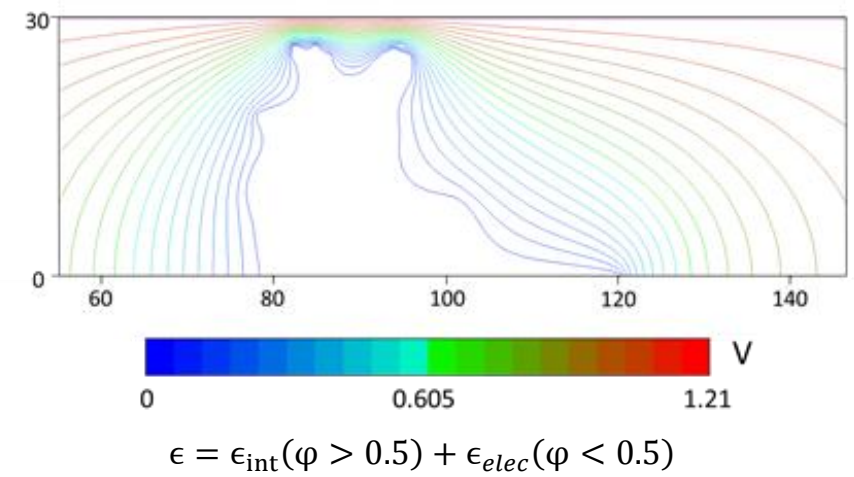

Figure 4. Electric Potential at time $\mathrm{t}=1.45 \mu \mathrm{s}$ obtained with the Poisson's equation (3) and clusters.

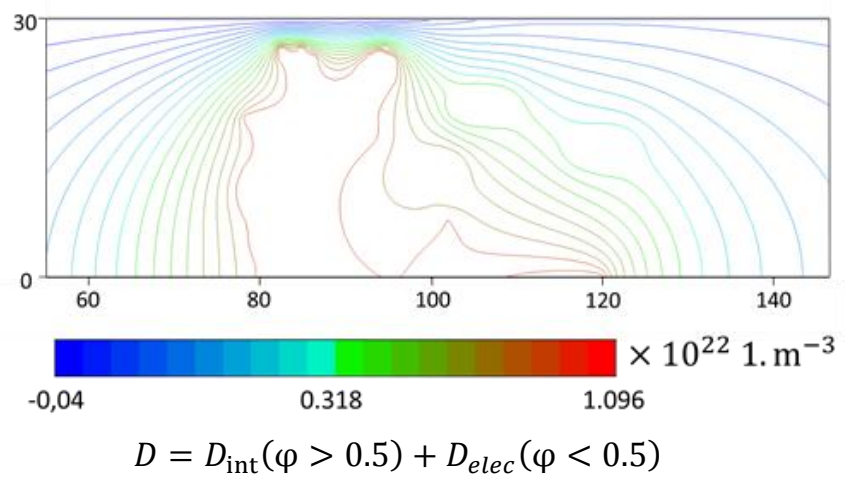

Figure 5. Cations concentration a time $\mathrm{t}=1.45 \mu \mathrm{s}$ obtained with the conservation law (2) and clusters.

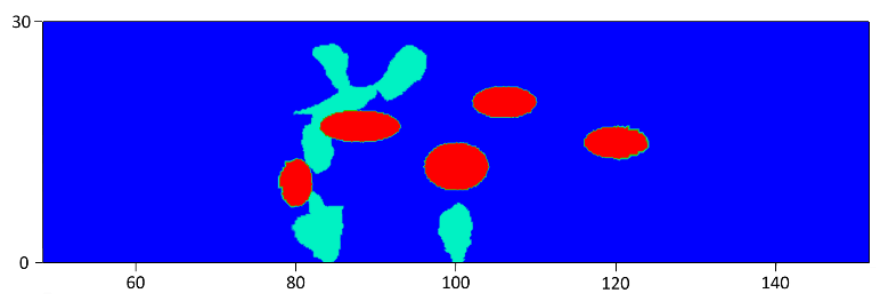

Figure 6. Results of filament growth with clusters in the electrolyte at time $\mathrm{t}=1.45 \mu \mathrm{s}$ 